Chinese Journal of Organic Chemistry

\title{
生物硫醇苂光探针的研究进展
}

\author{
陈莉 ${ }^{a}$ 黎俊波 ${ }^{b}$ 陈杜刚 $*, a$ \\ ( ${ }^{a}$ 武汉工程大学化工与制药学院 绿色化工过程教育部重点实验室 武汉 430205) \\ ( ${ }^{b}$ 武汉工程大学化学与环境工程学院 武汉 430205)
}

\begin{abstract}
摘要 生物硫醇(包含半胱氨酸、高半胱氨酸和谷胱甘肽)在生命活动中扮演了重要的角色, 其浓度的异常变化与某些疾 病息息相关，因此对硫醇的检测具有重要意义. 苂光探针因具有灵敏度高、时空分辨率好、无损伤、可视化等优势，在 生物硫醇的检测方面得到了高度重视. 利用硫醇在分子结构上的共同点(含統基的氨基酸)和差异(分子大小、亲核性、 空间位阻、细胞内含量), 可通过迈克尔加成、亲核芳基取代、加成环化等反应实现对硫醇的选择性检测. 综述了近 3 年来硫醇苂光探针领域的研究进展. 首先介绍了对硫醇有选择性识别的苂光探针, 随后分类讨论了对半胱氨酸、高半 胱氨酸和谷胱甘肽各具有特异性检测的荧光探针, 并重点介绍了分子设计、识别机理、苂光性质和成像应用, 初步探 讨了部分探针在监测细胞生命活动中的作用，同时还对本领域的发展提出了展望.
\end{abstract}

关键词 硫醇; 荧光探针; 半胱氨酸; 高半胱氨酸; 谷胱甘肽; 进展

\section{Recent Advances in Fluorescent Probes for Biothiols}

\author{
Chen, $\mathrm{Li}^{a} \quad \mathrm{Li}$ Junbo $^{b} \quad$ Chen, Dugang ${ }^{*, a}$ \\ (Key Laboratory for Green Chemical Process of Ministry of Education, School of Chemical Engineering and Pharmacy, \\ Wuhan Institute of Technology, Wuhan 430205) \\ (School of Chemistry and Environmental Engineering, Wuhan Institute of Technology, Wuhan 430205)
}

\begin{abstract}
Biothiols, including cysteine (Cys), homocysteine (Hcy) and glutathione (GSH), play an important role in life activities, and the fluctuation of their concentrations is closely related to many diseases. Therefore, it is of great importance to detect the content of biothiols. Among all varieties of techniques, the fluorescent probe demonstrates tremendous advantages ascribed to its good sensitivity, high temporal-spatial resolution, noninvasiveness and visualization. Taking advantage of the commonality and differences among the three thiols, namely, the similar reactive groups of sulfhydryl group and amino group, and the different molecular size, nucleophilicity, steric hindrance and intracellular contents, various recognition mechanisms, such as Michael addition, aromatic nucleophilic reaction and concerted addition and cyclization reaction, are all implemented to realize the specific detection of biothiols. The research progress of thiol fluorescent probes in recent three years is reviewed. The fluorescent probes that can selectively respond to biothiols as well as to Cys, Hcy and GSH are systemically analyzed and summarized. The molecular design, recognition mechanism, fluorescence properties and imaging applications are emphasized,, and the application of biothiol fluorescent probe on monitoring life activities is preliminary introduced. In addition, a perspective of future development in this area is also presented.
\end{abstract}

Keywords biothiol; fluorescent probe; cysteine; homocysteine; glutathione; advance

半胱氨酸(Cys)、高半胱氨酸(Hcy)和谷胱甘肽(GSH) 是广泛存在于细胞内的重要小分子硫醇氨基酸, 它们不 仅参与人体内各种生命活动, 在维持生物体系的氧化还 原稳态和蛋白质生物学构象方面起着至关重要的作 用 ${ }^{[1]}$, 而且还参与可逆氧化还原反应、信号转导、细胞
调亡和细胞增殖等过程，有利于调节新陈代谢和维持细 胞内稳态 ${ }^{[2-3]}$. 其中, Cys 不仅是谷胱甘肽, 乙酰辅酶和 牛磺酸的前体化合物, 而且是生物硫铁配合物中硫配体 的提供者 ${ }^{[4-7]}$. Hcy 是由蛋氨酸的去甲酰化作用形成的, 属于蛋氨酸循环的中间产物 ${ }^{[8-9]}$. 它主要来自饮食中蛋

\footnotetext{
* Corresponding author. E-mail: dg.chen@wit.edu.cn

Received June 23, 2020; revised July 27, 2020; published online September 15, 2020.

Project supported by the National Natural Science Foundation of China (No. 51703171), and the Graduate Innovative Fund of Wuhan Institute of Technology (No. CX2019003)

国家自然科学基金(No. 51703171)、武汉工程大学研究生教育创新基金(No. CX2019003)资助项目.
} 
氨酸的摄入，通常通过与 Cys 残基的二硫键或赖氨酸 (Lys)残基的酰胺键在翻译后整合到蛋白质中合成 ${ }^{[10-11]}$. GSH 是一种 $\gamma$-酰胺键, 是由谷氨酸、半胱氨酸和甘氨酸 组成的含硫醇的三肽, 几乎存在于人体的每个细胞中 (图 1) ${ }^{[12-14]}$. 生物体内 Cys、Hcy 和 GSH 的含量分别为 $30 \sim 200 、 5 \sim 12$ 和 $1 \sim 10 \mathrm{mmol} \cdot \mathrm{L}^{-1}$, 其中 $\mathrm{GSH}$ 相对于 Cys 和 Hcy 高很多, 它们在细胞内的含量变化与体内多 种疾病息息相关 ${ }^{[15-20]}$. 当 Cys 过多或过少时都会引起儿 童生长缓慢、嗜睡、皮肤病、脱发、肝脏损害、肌肉松 弛和身体肥胖衰竭等疾病 ${ }^{[21-25]}$. 健康成年人体内 Hcy 正 常水平在 $9 \sim 13 \mu \mathrm{mol} \cdot \mathrm{L}^{-1}$ 范围内, 血清中 Hcy 的异常高 (超过 $15 \mu \mathrm{mol} \cdot \mathrm{L}^{-1}$ ) 会造成先天性缺陷和认知功能障碍, 也会造成心血管疾病、阿尔兹海默症等 ${ }^{[26-28]}$. GSH 作为 细胞内含量最丰富的生物硫醇, 它的浓度与多种细胞功 能相关, 如生长、代谢以及对癌症的放疗和化疗的耐药 性等 ${ }^{[29-33]}$. 因此实现对生物体内硫醇氨基酸的准确检测 对于疾病的评估和诊断非常重要.<smiles>NC(CS)C(=O)O</smiles><smiles>NC(CCSCCO)C(=O)O</smiles><smiles>NC(CCC(=O)NC(CS)C(=O)NCC(=O)O)C(=O)O</smiles>

图 1 三种生物硫醇的化学结构

Figure 1 Molecular structures of three biothiols

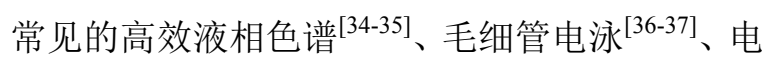

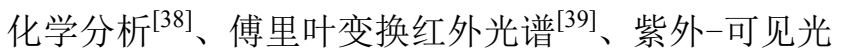
谱和苂光光谱 ${ }^{[40-41]}$ 等方法已被广泛应用于硫醇氨基 酸的检测. 在众多方法中, 苂光探针由于使用方便、 损害性小、灵敏度高等优势受到了广泛的关注 ${ }^{[42-47]}$. 苂 光探针一般是由识别基团、荧光基团和连接体部分组成 (图 2), 与目标分析物接触时会触发探针苂光信号的变 化, 从而实现对分析物的定性或定量检测. 基于荧光探 针的苂光显微法可以提供高的时空分辨率和可视化成 像, 在生物化学和医学研究上具有重要意义 ${ }^{[48-49]}$.

根据硫醇分子的结构特征和反应活性, 可以通过迈 克尔加成、加成环化、亲核取代等多种反应机理来设计 各种对硫醇具有选择性的荧光探针 ${ }^{[50]}$. 本文以检测的 对象为基准, 对近 3 年来报道的部分硫醇荧光探针进行 分类总结, 分别介绍了普通硫醇荧光探针和对单一硫醇 (Cys, Hcy 和 GSH) 有特异性识别能力的苂光探针, 并对 其分子设计、荧光性质、检测机理和生物应用加以讨论.

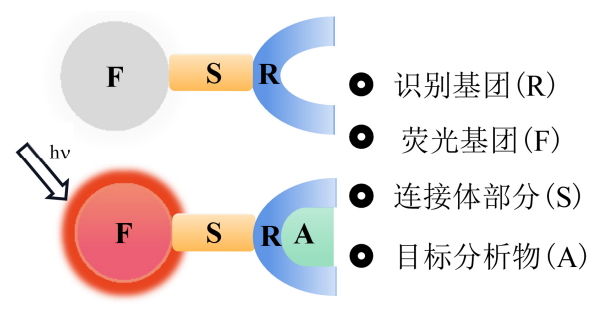

图 2 苂光探针示意图

Figure 2 Schematic illustration of the fluorescent probe

\section{1 生物硫醇荧光探针}

\section{1 普通硫醇荧光探针}

Cys, Hcy 和 GSH 在结构上都具有反应活性较高的 颈基，因此，可以利用颈基的亲核性与二硫化物、磺酰 胺、磺酸酯或不饱和双键等发生可逆或不可逆的反应来 设计硫醇荧光探针 ${ }^{[26]}$. 当探针与硫醇发生反应后, 光物 理性质会发生改变，如荧光开启或不同波长处苂光强度 的比率发生变化, 从而实现对硫醇的特异性检测.

陈杜刚等 ${ }^{[51]}$ 报道了一种以 2,4-二硝基苯磺酰基 (DNBS)为识别位点的具有较大斯托克斯(Stokes)位移、 发射深红光的苂光探针 1 (如图 3). 由于 DNBS 的强缺 电子特性导致分子内的光致电荷转移(PET)效应, 从而 使探针分子的苂光淬灭. 向探针所在的乙醇和 4-羟乙基 哌嗪乙磺酸(HEPES)缓冲液 $(V / V=1 / 1, \mathrm{pH}=7.4)$ 中加入 硫醇时，颈基与 DNBS 发生亲核芳香取代反应，使其从 分子中断裂，从而生成苂光团 $\mathbf{1 a}$ 并开启荧光，吸收峰从 $420 \mathrm{~nm}$ 红移到 $480 \mathrm{~nm}$ 处, 发射峰为 $640 \mathrm{~nm}$. 其中, Cys 对探针溶液的响应速度最快. 探针对 Cys、Hcy 和 GSH 的检测限分别为 $0.11 、 0.47$ 和 $0.35 \mu \mathrm{mol} \cdot \mathrm{L}^{-1}$. 目前该探 针已被成功应用于 MCF-7 细胞中内源性生物硫醇的苂 光成像.

同样以 DNBS 为识别位点的苂光探针还有 2,3 和 4 (图 3). 2019 年, 宋相志等 ${ }^{[52]}$ 以甲基化喹啉衍生物为荧光 团构建了水溶性的苂光探针 2. 识别硫醇后在 $613 \mathrm{~nm}$ 处 发出强烈的红色荧光, 斯托克斯位移高达 $115 \mathrm{~nm}$. 王雅 雯等 ${ }^{[53]}$ 利用荧光素衍生物为骨架, 合成了两个开启型 的荧光探针 3 和 $\mathbf{4}$, 成功应用于 HeLa 细胞中硫醇的荧光 成像.

阴彩霞等 ${ }^{[54]}$ 以硝基苯并恶二唑(NBD)为识别基团 合成了可以区分 $\mathrm{Cys} / \mathrm{Hcy}$ 和 GSH 的双通道荧光探针 5 (图 4). 因 NBD 的强缺电子特性, 导致的 PET 效应使探 针分子的荧光淬灭. 在探针所在的 $\mathrm{PBS}$ 和 $\mathrm{CH}_{3} \mathrm{CN}$ 的混 合溶液 $(\mathrm{pH}=7.4, V / V=8 / 2)$ 中加入硫醇时, 会发生亲核 取代反应，醚键断裂，从而生成琉基取代的 NBD 片段 和化合物 $5 \mathbf{a}$, 发射波长为 $625 \mathrm{~nm}$. 当所加入的硫醇种类 不同时，所生成 NBD 片段的结构也不一样，导致其 
<smiles>CC1(C)OC(C(C#N)=C2CCCCC2)=C(C#N)/C1=C\c1ccc2cc(OS(=O)(=O)c3ccc([N+](=O)[O-])cc3[N+](=O)[O-])ccc2c1</smiles><smiles>CC1(C)OC(=C(C#N)C#N)C(C#N)=C1C=Cc1ccc2cc(O)ccc2c1</smiles><smiles></smiles><smiles>O=Cc1c(OS(=O)(=O)c2ccc([N+](=O)[O-])cc2[N+](=O)[O-])ccc2c1Oc1cc(OS(=O)(=O)c3ccc([N+](=O)[O-])cc3[N+](=O)[O-])ccc1C21OC(=O)c2ccccc21</smiles>

3<smiles>N#Cc1c(OS(=O)(=O)c2ccc([N+](=O)[O-])cc2[N+](=O)[O-])ccc2c1Oc1cc(OS(=O)(=O)c3ccc([N+](=O)[O-])cc3[N+](=O)[O-])ccc1C21OC(=O)c2ccccc21</smiles>

图 3 普通硫醇苂光探针 $1 \sim 4$

Figure 3 Common biothiol fluorescent probes $\mathbf{1} \sim \mathbf{4}$

发光状态有明显差别. 当加入的硫醇是 Cys/Hcy 时, 分 子内的氨基和巯基进一步发生重排反应释放出新的荧 光团 $\mathbf{5 b}$, 其发射波长为 $550 \mathrm{~nm}$, 从而表现出红光和绿 光的双通道荧光发射的性质, 对 $\mathrm{Cys} / \mathrm{Hcy}$ 的检测限为
$0.061 / 0.048 \mu \mathrm{mol} \cdot \mathrm{L}^{-1}$. 当加入 $\mathrm{GSH}$ 时, 由于位阻效应不 能诱导发生分子内的重排反应，由于 NBD 片段的结构 为不发荧光的 $\mathbf{5 c}$, 因此只表现出 $\mathbf{5 a}$ 的单一红光信号, 检测限为 $0.082 \mu \mathrm{mol} \cdot \mathrm{L}^{-1}$. 探针已成功用于检测拟南芥 中的硫醇, 这对于研究植物中硫醇的转化和代谢途径具 有重要意义.

刘伟生等 ${ }^{[5]}$ 以香豆素和半花青素为苂光团合成了具 有三个反应位点的苂光探针 6(图 4). 探针结构中引入了 三个反应位点以区别生物硫醇的荧光信号, 引入了半菁 结构以实现近红外(NIR)苂光发射, 引入了烷基链离子 以提高水溶性和生物相容性. 向探针所在 $\mathrm{PBS}(\mathrm{pH}=$ $7.4,10 \mathrm{mmol} \cdot \mathrm{L}^{-1}$ ) 缓冲溶液中, 加入浓度为 $0 \sim 250$ $\mathrm{mmol} \cdot \mathrm{L}^{-1}$ 的硫醇进行检测. 添加 Cys 时, 在 $60 \mathrm{~min}$ 内 $586 \mathrm{~nm}$ 处的吸收逐渐减弱, 并在 518 和 $419 \mathrm{~nm}$ 处出现 两个新的吸收峰; 添加 Hcy 时, 在 $90 \mathrm{~min}$ 内 514 和 418 $\mathrm{nm}$ 处出现两个吸收峰; 添加 GSH 时, 在 $70 \mathrm{~min}$ 内 586 $\mathrm{nm}$ 处的吸收峰缓慢下降, 同时在 $412 \mathrm{~nm}$ 处出现新的吸 收峰. 当激发波长为 $419 \mathrm{~nm}$ 时, 探针在 $498 \mathrm{~nm}$ 处的荧 光发射强度急剧增强, 并且 Cys/Hcy 诱导的苂光信号比 GSH 强; 以 $518 \mathrm{~nm}$ 激发后, 探针分别在 573、616、727 和 $783 \mathrm{~nm}$ 处显示 4 个苂光发射; 当连续添加 $\mathrm{Cys} / \mathrm{Hcy} /$ GSH 时, 在 573 和 $616 \mathrm{~nm}$ 处的荧光强度显著增加, 而在 727 和 $783 \mathrm{~nm}$ 处严重降低; 以 $555 \mathrm{~nm}$ 为激发波长时，探 针在 612 和 $727 \mathrm{~nm}$ 处出现两个苂光发射. 检测限分别为 2.2 (Cys)、2.08 (Hcy) 和 $1.89(\mathrm{GSH}) \mathrm{mmol} \cdot \mathrm{L}^{-1}$. 探针通过 三个不同的发射通道同时对活细胞中的外源性和内源 性生物硫醇成像，从而促进人们对复杂的生理和病理过 程中硫醇的产生、作用机理和代谢过程的了解, 并有助 于预防和诊断与生物硫醇有关的疾病.

2001 年，唐本忠等 ${ }^{[55]}$ 首次提出了聚集诱导发光 (AIE)的概念, 巧妙地克服了传统荧光探针在水溶液体
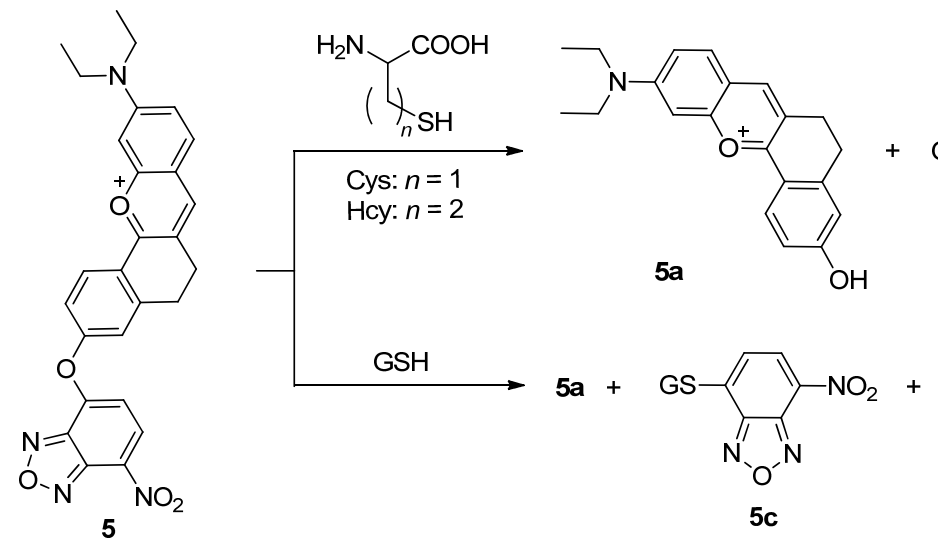

图 4 普通硫醇荧光探针 5 和 6

Figure 4 Common biothiol fluorescent Probes 5 and 6 
系内容易发生聚集诱导苂光淬灭(ACQ)的问题 ${ }^{[56]}$. 霍延 平等 ${ }^{\left[{ }^{[} 7\right]}$ 将四苯乙烯衍生物作为荧光基团, 将马来酰亚 胺作为识别基团, 设计并合成了两个能特异性检测 Cys/Hcy 的 AIE 荧光探针 7 和 8(图 5). 因 PET 作用, 马 来酰亚胺会淬灭探针的苂光. 当向探针所在 $\mathrm{H}_{2} \mathrm{O}$ 与 $\mathrm{THF}$ 的混合溶液 $(V / V=99 / 1)$ 中加入 Cys/Hcy 后, 颈基会与马 来酰亚胺发生加成反应, 阻断 PET 作用使四苯乙烯衍生 物荧光恢复, 发射波长为 $530 \mathrm{~nm}$, 且荧光强度增强了 2000 倍. 但加入 GSH 后, 由于 GSH 本身的空间阻碍效 应, 反应几乎不发生, 因此无法开启探针的苂光. 目前 这些探针已经成功应用于 H1299 肺癌细胞中生物硫醇 的检测.

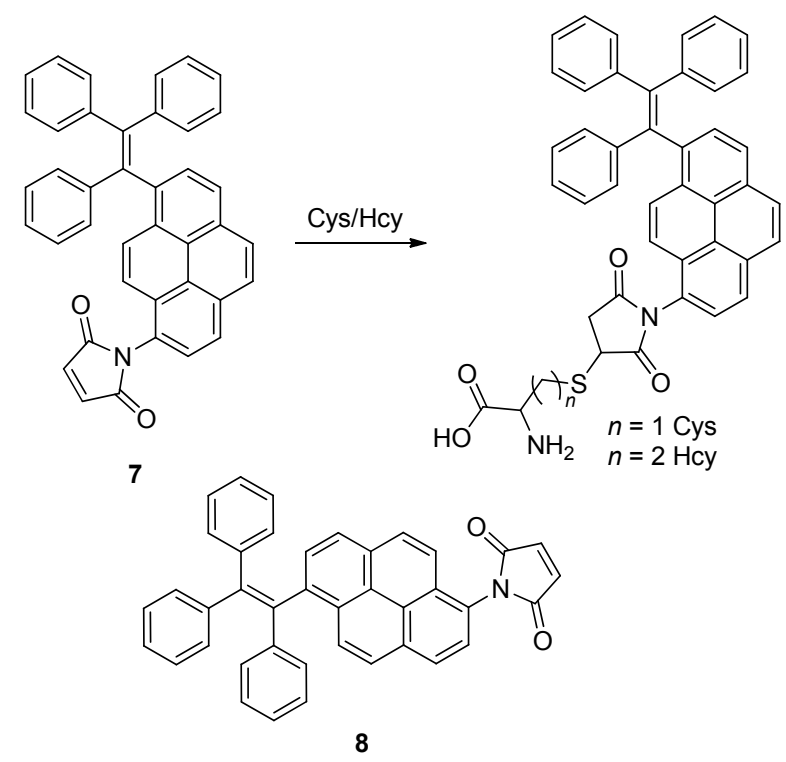

图 5 普通硫醇荧光探针 7 和 8

Figure 5 Common biothiol fluorescent probes $\mathbf{7}$ and $\mathbf{8}$

上述荧光探针对三种硫醇的特异性识别效果并不 是太好, 最多只能对两种硫醇(Cys/Hcy)表现出选择性 响应，而不能有针对性地检测某一种特定的硫醇. 它们 的出现对硫醇分子反应活性的研究和探针分子的设计 做出了贡献，接下来我们将分别讨论对 Cys、Hcy 和 GSH 具有特异性识别功能的各种硫醇苂光探针.

\section{2 半胱氨酸特异性荧光探针}

半胱氨酸(Cys)是一种蛋白质生成性的必需氨基酸, 也是 20 多种天然氨基酸中唯一含颈基小分子的氨基酸. 它广泛存在于细胞中, 参与肽链的合成和折叠, 在折叠 的蛋白质中提供二硫键来稳定蛋白质结构以增加其刚 性并发挥其适当的功能, 同时参与氧化还原平衡, 并调 节细胞信号传导、排毒、细胞调亡和许多其它生命过程, 在生物系统中具有不可或缺的地位 ${ }^{[88]}$. 因此, 利用荧光 探针在生物体内实现对 Cys 的特异性检测具有重要的意 义. 当前应用于特异性识别 Cys 的方法主要有: 与醛基
的环化反应、迈克尔加成反应、丙烯酸酯的环化脱除反 应和芳香取代重排反应等.

\subsection{1 醛环化反应}

由于半胱氨酸的氨基和颈基可以先后与醛基发生 反应生成噻唑烷, 从而改变化合物的荧光性质. 因此, 基于醛环化反应的机理被广泛应用于半胱氨酸特异性 苂光探针的开发 ${ }^{[26]}$.

张友玉等 ${ }^{\left[{ }^{[99}\right.}$ 设计了一种具有荧光和比色的双通道 探针 9(图 6), 用来检测 Cys. 在探针的 DMF 和 PBS 混 合溶液 $\left(V / V=1 / 1,10 \mathrm{mmol} \cdot \mathrm{L}^{-1}, \mathrm{pH}=7.4\right)$ 中加入 $\mathrm{Cys}$ 后, Cys 的胺基与探针的醛基反应生成亚胺，其进一步与颈 基发生加成反应, 在 $15 \mathrm{~min}$ 内生成具有较弱共轭作用的 五元噻唑烷 9a, 其发射波长为 $440 \mathrm{~nm}$, 荧光强度显著增 强(量子产率从 $0.67 \%$ 增强到 $13.63 \%$ ), 且溶液由黄色变 为无色, 检测限为 $63 \mathrm{nmol} \cdot \mathrm{L}^{-1}$. 当向探针溶液中加入 $\mathrm{Hcy} / \mathrm{GSH}$ ，几乎没有显现苂光响应，当 Hcy/GSH 加入到 有 $\mathrm{Cys}$ 的探针溶液中时, 苂光光谱在 $50 \mathrm{~min}$ 内与仅加入 Cys 时所产生的苂光几乎保持一致, 探针溶液颜色并未 发生任何变化, 表明探针对于 Cys 的选择性明显高于 Hcy/GSH. 因此, 可利用比色法区分这三种重要硫醇. 另外，比色和苂光双信号可以进一步确保检测的准确 性. 探针目前已成功应用于人体血清细胞中 Cys 的检测.

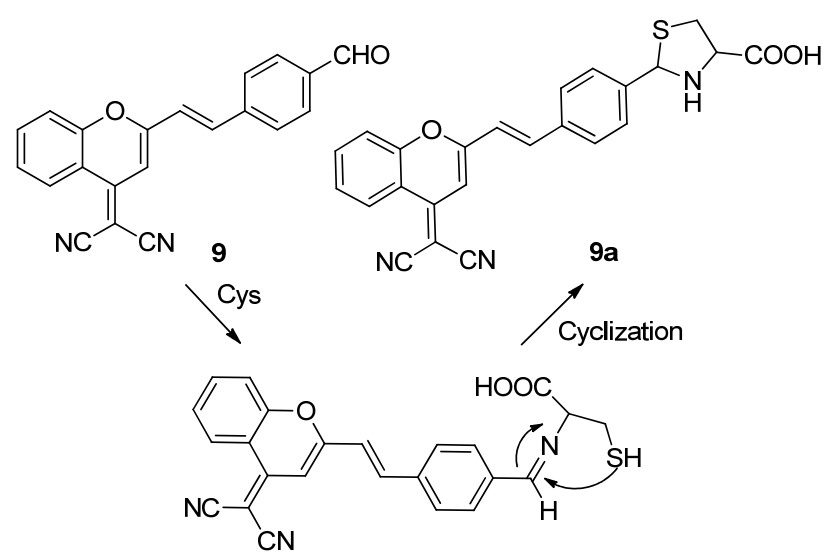

图 6 以醛基为识别位点的 Cys 苂光探针 9

Figure 6 Probe 9 with aldehyde as the recognition site for Cys detection

彭羽等 ${ }^{\left[{ }^{[0]}\right.}$ 以丙烯酸酯和醛基为识别基团制备了具 有双重响应位点的开启型荧光探针 $\mathbf{1 0}$ (图 7). 在探针 $\mathbf{1 0}$ 的 HEPES 缓冲溶液 $\left(\mathrm{pH}=6.8,37{ }^{\circ} \mathrm{C}\right)$ 中加入 Cys 后, 它 能在 $5 \mathrm{~s}$ 内与丙烯酸酯部分发生协同加成反应，生成内 酰亚胺并从探针分子中断裂，与此同时还能与醛基发生 环化反应形成五元噻唑烷，从而得到产物 $10 \mathrm{a}$, 发射峰 为 $525 \mathrm{~nm}$, 荧光增强且溶液从无色变成强绿色, 检测限 为 $61.7 \mathrm{nmol} \cdot \mathrm{L}^{-1}$. 与 $\mathrm{Hcy}$ 和 $\mathrm{GSH}$ 相比, Cys 可以与探针 反应形成有利的七元环, 从而将 Cys 与 Hcy 和 GSH 区 
别开.<smiles>C=CC(=O)Oc1ccc2c(c1)Oc1c(ccc(O)c1C=O)C21OC(=O)c2ccccc21</smiles><smiles>[R]c1ccc(C(=Cc2ccc(C(=C(c3ccc([R])cc3)c3ccc(C=CC=O)cc3)c3ccc([R])cc3)cc2)c2ccc(C3CC=NC(C(=O)O)C3)cc2)cc1</smiles><smiles>[R]c1ccc(C(=C(c2ccc(C3CC(SCC(N)C(=O)O)NC(C(=O)O)C3)cc2)c2ccc(C3SCC(C(=O)O)NC3SCCC(N)C(=O)O)cc2)c2ccc([R])cc2)cc1</smiles>

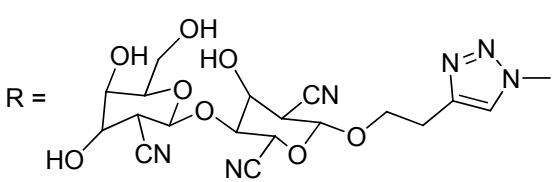

图 7 以醛基为识别位点的 Cys 苂光探针 10 和 11

Figure 7 Probes $\mathbf{1 0}$ and $\mathbf{1 1}$ with aldehyde as the recognition site for Cys detection

张媛等 ${ }^{[61]}$ 以 $\alpha, \beta$-不饱和醛为识别基团, 以乳糖取代 的四苯乙烯为苂光基团, 合成了 AIE 型比率荧光探针 $\mathbf{1 1}$ (图 7). 两亲性的探针 11 能在 PBS 缓冲溶液 $(67 \mathrm{mmol} \cdot$ $\left.\mathrm{L}^{-1}, \mathrm{pH}=7.4\right)$ 中自组装成纳米颗粒, 添加的 $\mathrm{Cys}$ 能与探 针的 $\alpha, \beta$-不饱和醛发生成环反应生成化合物 11a, 而 11a 能与过量的 Cys 进一步发生亲核加成反应生成水溶性更 好的产物 11b, 在此反应过程中荧光发射波长从 $575 \mathrm{~nm}$ 蓝移至 $500 \mathrm{~nm}$, 苂光强度比 $\left(I_{500} / I_{575}\right)$ 从 0.48 增加到 1.76 ,
检测限为 $25 \mu \mathrm{mol} \cdot \mathrm{L}^{-1}$. 而 Hcy 的加入仅能引起微小变 化, 即强度比 $\left(I_{500} / I_{575}\right)$ 从 0.51 略微增加到 $0.79 ; \mathrm{GSH}$ 的 加入从 0.53 到 0.54 几乎没有波动, 从而将这三种硫醇区 别开来.

\subsection{2 迈克尔加成反应}

自从 Chakraborti 及其同事发现硫醇能在水中与 $\alpha, \beta$ 不饱和酮之间发生迈克尔加成反应，生成硫醚，之后研 究人员 ${ }^{[62-65]}$ 通过该反应机理报道了一系列硫醇苂光探 针.

李敏娜等 ${ }^{[6]}$ 合成了以 $\alpha, \beta$-不饱和酮取代的钓(II)配 合物 $\left(\mathrm{Ru}(\mathrm{bpy})_{3}^{2+}\right)$ 荧光探针 12(图 8). 由于联吡啶和钓的 配合物到不饱和酮单元的分子内电荷转移(ICT) 效应, 导致探针在 HEPES 的缓冲溶液 $\left(10 \mathrm{mmol} \cdot \mathrm{L}^{-1}, \mathrm{pH}=7.4\right)$ 中基本不发荧光, 加入 Cys 后, 它与 $\alpha, \beta$-不饱和酮部分 发生 1,2-加成反应，从而破坏酮和吡啶之间的 $\pi-\pi$ 共轭， 使 ICT 终止, 在 $10 \mathrm{~min}$ 内生成产物 12a. 以 $470 \mathrm{~nm}$ 为激 发波长, 在 $620 \mathrm{~nm}$ 处有显著的红色荧光发射, 荧光强度 增加 30 倍, Stokes 位移为 $150 \mathrm{~nm}$, 检测限为 $3.2 \mu \mathrm{mol} \cdot$ $\mathrm{L}^{-1}$. 该探针成功地应用于骨间充质干细胞(BMSCs)和 斑马鱼中内源性硫醇的成像, 也能应用于生物学中检测 新生牛血清中的硫醇, 对促进生物硫醇的生理和病理学 研究具有重要意义. 在 Hcy 和 GSH 存在的情况下, 25 min 内苂光强度几乎没有发生变化. 探针对 Cys 表现出 高敏感性的原因，一方面是由于 Cys 的空间位阻低于 GSH 和 Hcy, 另一方面是由于其强带正电的探针和带负 电的 Cys 之间的静电引力低于 Hcy 和 GSH.

王巍等 ${ }^{[67]}$ 报道了一种基于 PET/ICT 双淬灭机制的 双光子开启型苂光探针 13(图 8), 其中，马来酰亚胺基 团具有 PET 淬灭效应, 羰基吸电子基团能引发 ICT 效 应. 由于双淬灭作用，探针在溶液中不发荧光，而加入 的 Cys 与马来酰亚胺部分会发生迈克尔加成和环化级联 反应, 在 $2 \mathrm{~min}$ 内生成中间体 13a, $30 \mathrm{~min}$ 内生成产物 13b, 在 $446 \mathrm{~nm}$ 处开启苂光且苂光强度增强 3000 倍，检 测限为 $1.4 \mathrm{nmol} \cdot \mathrm{L}^{-1}$, 苂光量子产率为 0.782 . 探针被成 功用于 $\mathrm{HeLa}$ 细胞中 Cys 的双光子成像.

\subsection{3 协同加成环化反应}

Strongin 小组 ${ }^{[26]}$ 报道了利用丙烯酸酯加成环化反应 来特异性检测 Cys, 之后研究人员以各种苂光团, 如香 豆素、BODIPY、罗丹明等, 构建了一系列对 Cys 具有 良好选择性的苂光探针.

王毅等 ${ }^{[68]}$ 合成了一种以双丙烯酰基为识别基团的 荧光探针 14(图 9). 由于在双丙烯酰基的 PET 作用下, 探针在 DMSO 与 HEPES 的缓冲溶液 $(V / V=7: 3, \mathrm{pH}=$ 7.4)中基本不发荧光，而加入的 Cys 能与探针发生协同 迈克尔加成和分子内成环反应, 裂解出内酰亚胺, 从而 


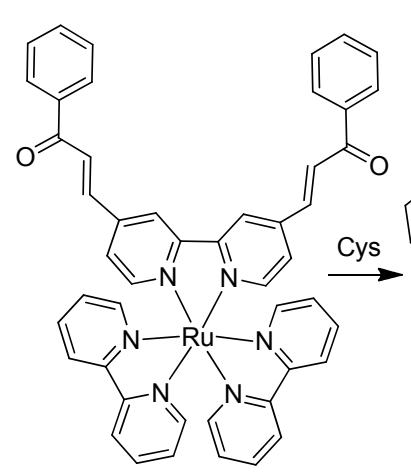

12
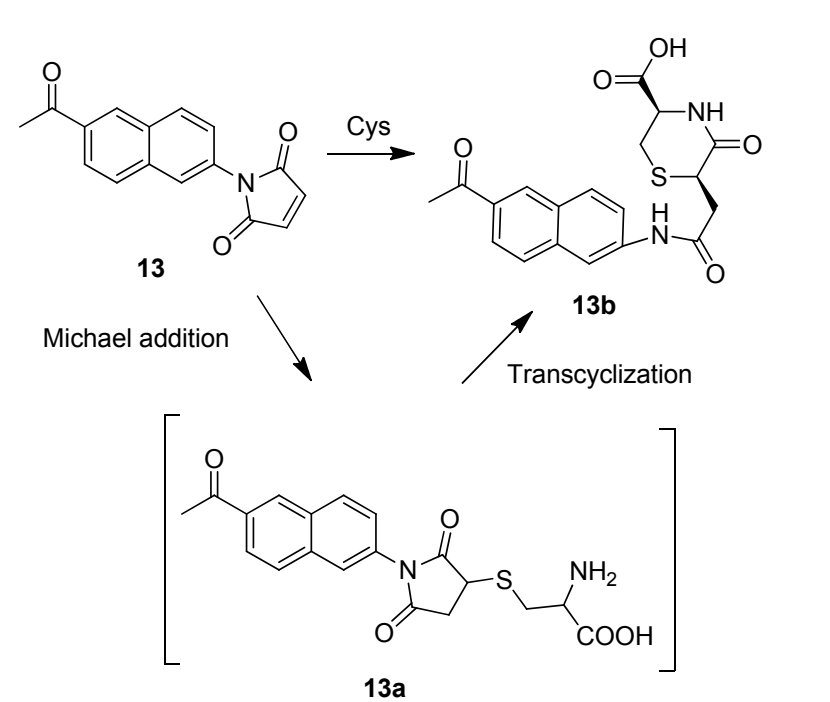

图 8 迈克尔加成检测 Cys 的探针 12 和 $\mathbf{1 3}$

Figure 8 Probes $\mathbf{1 2}$ and $\mathbf{1 3}$ based on Michael addition mechanism for Cys detection

释放出苂光团 14a. 其中, 14a 的羟基和氮原子能进一步 引发激发态的分子内电荷转移(ESIPT)效应, 发射波长 为 387 和 $470 \mathrm{~nm}$, 苂光强度增强 4725 倍, 检测限为 32.6 $\mathrm{nmol} \cdot \mathrm{L}^{-1}$. 当 Hcy 加入后会使荧光略微变化, 但荧光强 度变化值相比于 Cys 与探针反应后荧光强度的增强倍 数, 可忽略, 而其他氨基酸的加入几乎不会引起苂光信 号的变化. 该探针已成功用于 HepG2 细胞和斑马鱼中 的 Cys 成像. 同样以丙烯酰基为识别基团, 基于 ESIPT 机理的 Cys 苂光探针有丁彩凤等 ${ }^{[69]}$ 合成的荧光探针 15 (图 9), 发射波长为 $595 \mathrm{~nm}$, 检测限为 $19 \mathrm{nmol} \cdot \mathrm{L}^{-1}$; 韩 翠艳等 ${ }^{[70]}$ 设计的荧光探针 16(图 9), 发射波长为 $640 \mathrm{~nm}$, 检测限为 $55 \mathrm{nmol} \cdot \mathrm{L}^{-1}$.

文献中还报道了许多以丙烯酸酯为识别基团选择 性检测 Cys 的红光或 NIR 苂光探针. 如范小林等 ${ }^{[71]}$ 合成 的探针 17(图 10), 识别 Cys 后, 发射波长为 $741 \mathrm{~nm}$, 检 测限为 $210 \mathrm{nmol} \cdot \mathrm{L}^{-1}$. 吕正亮等 ${ }^{[72]}$ 合成了探针 $\mathbf{1 8}$ (图 10), 能在 $1 \mathrm{~min}$ 内识别 Cys 并释放出荧光团, 发射波长为 645 $\mathrm{nm}$, 检测限为 $10 \mathrm{nmol} \cdot \mathrm{L}^{-1}$. 袁宇等 ${ }^{[73]}$ 合成了水溶性苂 光探针 19(图 10), 发射波长为 $674 \mathrm{~nm}$, 检测限为 0.96
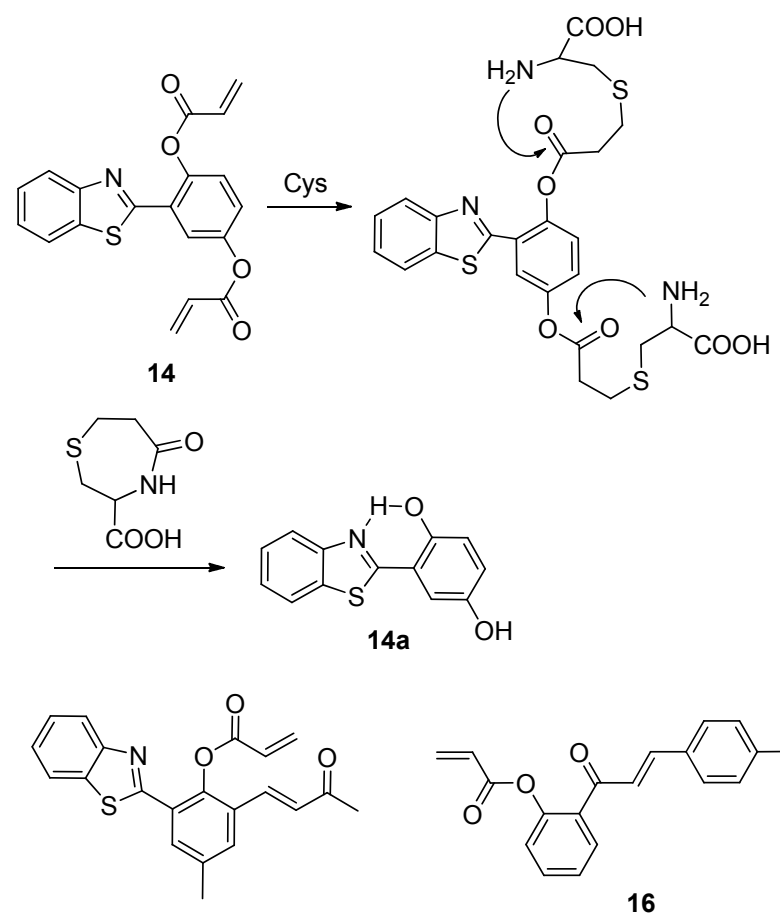

15

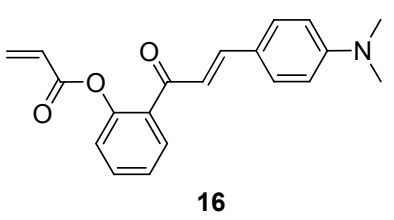

图 9 丙烯酸酯加成环化检测 Cys 的探针 14 16

Figure 9 Probes 14 16 based on addition-cyclization mechanism for Cys detection

$\mu \mathrm{mol} \cdot \mathrm{L}^{-1}$. 王炳祥等 ${ }^{[74]}$ 以二氰基异佛尔酮为苂光团合成 了探针 20 (图 10), 发射波长为 $673 \mathrm{~nm}$, 检测限为 0.16 $\mu \mathrm{mol} \cdot \mathrm{L}^{-1}$. 与探针 20 类似的有江玉亮等 ${ }^{[1]}$ 报道的探针 21 (图 10), 发射波长为 $631 \mathrm{~nm}$, 检测限为 $0.26 \mu \mathrm{mol} \cdot \mathrm{L}^{-1}$. 祁争健等 ${ }^{[75]}$ 设计合成了荧光探针 22 (图 10), 发射波长为 $770 \mathrm{~nm}$, 检测限为 $0.1323 \mu \mathrm{mol} \cdot \mathrm{L}^{-1}$, 该探针能够通过直 观的肉眼观测溶液颜色的变化, 有效区分水溶液中的 Cys、Hcy 和 GSH. 陈美等 ${ }^{[76]}$ 以花青素衍生物为荧光基 才的双光子(TP)苂光探针 23(图 10), 识别 Cys 后在 611 $\mathrm{nm}$ 处发射苂光，苂光增强 51 倍，检测限为 $24.1 \mathrm{nmol}$ $\mathrm{L}^{-1}$. 陈杜刚等 ${ }^{[77]}$ 报道了一种以姜黄素衍生物为荧光团 的 D-A-D 型苂光探针 24(图 10), 为了降低探针的背景信 号, 在分子的两端给体上各连接一个丙烯酸酯基团, 通 过 PET 作用有效淬灭探针的荧光. 识别 Cys 后发射波长 为 $615 \mathrm{~nm}$, 苂光增强 11 倍, 检测限为 $1.55 \mu \mathrm{mol} \cdot \mathrm{L}^{-1}$.

除此之外, 还有一些荧光探针能定位检测细胞器内 Cys. 麻秋娟等 ${ }^{[78]}$ 以 1,8-䒬二甲酰亚胺衍生物为苂光基 才，以 4-(2-氨基乙基)吗啉单元为溶酶体靶向基团合成 了开启型苂光探针 25(图 10), 探针能选择性地在细胞的 溶酶体内富集，识别 Cys 后，发射波长为 $550 \mathrm{~nm}$, 检测 限为 $0.069 \mu \mathrm{mol} \cdot \mathrm{L}^{-1}$.

麻秋娟等 ${ }^{[79]}$ 以香豆素衍生物为能量供体、罗丹明衍 生物为能量受体合成了基于苂光共振能量转移(FRET) 的比率型苂光探针 26(图 11). 在探针溶液中, 罗丹明部 

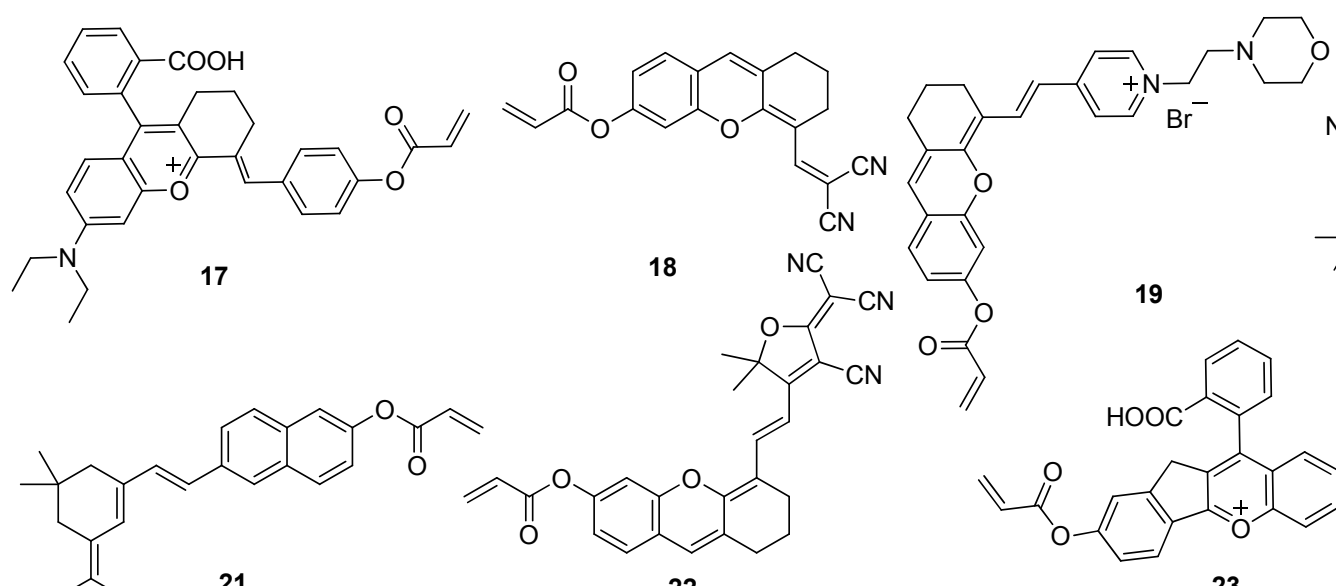

19<smiles>C=CC(=O)Oc1ccc(/C=C/C2=CC(=C(C#N)C#N)CC(C)(C)C2)cc1</smiles><smiles>C=CC(=O)Oc1ccc2cc(/C=C/C3=CC(=C(C#N)C#N)CC(C)(C)C3)ccc2c1</smiles><smiles></smiles>

20<smiles>C=CC(=O)Oc1ccc2c3c(cccc13)C(=O)N(CCN1CCOCC1)C2=O</smiles>

图 10 丙烯酸酯加成环化检测 Cys 的探针 $17 \sim 25$

Figure 10 Probes 17 $\sim \mathbf{2 5}$ based on addition-cyclization mechanism for Cys detection

分处于非荧光的螺内酯状态，使 FRET 过程受到抑制， 故在 $470 \mathrm{~nm}$ 处呈现出香豆素发色团的蓝色荧光; 识别 Cys 后, 丙烯酸酯部分从分子中脱除生成 $\mathbf{2 6 b}$, 并开启 了从香豆素到罗丹明的 FRET 效应, 发射波长为 543 $\mathrm{nm}$, 检测限为 $20 \mu \mathrm{mol} \cdot \mathrm{L}^{-1}$.

\subsection{4 芳香取代重排反应}

由于 Cys 包含巯基和氨基两个活性基团, 且两个基 团间距离很近, 当颈基发生亲核取代反应后, 氨基可进 一步与䘪基发生交换反应，从而改变分子的苂光性质， 实现对 Cys 的选择性检测 ${ }^{[80]}$.

周莹等 ${ }^{[81]}$ 合成了以芳基氯为识别基团的荧光探针 27(图 12). 向探针溶液中加入 Cys 后, 巯基与氯原子发 生芳香亲核取代反应生成中间产物 $27 \mathrm{a}$, 当 Cys 的氨基 与巯基交换后, 巯基能进一步与缺电子的双键发生加成 反应生成含七元环的化合物 $\mathbf{2 7 b}$, 反应过程中发射波长 蓝移 $200 \mathrm{~nm}$ 至 $456 \mathrm{~nm}$, 且苂光增强 20 倍, 检测限为 $0.463 \mathrm{nmol} \cdot \mathrm{L}^{-1}$. Hcy 与探针反应后, 由于分子结构的差 异, 不能与巯基发生加成反应成环; GSH 与探针发生芳 香亲核取代反应时会产生中间产物. 由于氨基香豆素的 十元环过渡态不稳定, 导致 GSH 很难与探针进一步发 生分子内重排反应, 因此 Hcy 和 GSH 的存在不会干扰 探针对 Cys 的检测.

郭炜等 ${ }^{[82]}$ 合成了苂光探针 28(图 13), 它是以硅-罗 丹明为荧光团, 以 4-甲氧基硫代苯酚作为识别基团, 因 PET 效应, 探针本身的荧光很弱. 在 $2 \mathrm{~min}$ 内, Cys 能与
探针通过连续的亲核芳香取代和分子内重排反应生成 苂光团 28a, 探针分子在 $688 \mathrm{~nm}$ 处的吸收峰消失，同时 在 $472 \mathrm{~nm}$ 处出现新的发射峰, $620 \mathrm{~nm}$ 处苂光发射增强; 与 GSH 反应后, 能在 $1 \mathrm{~min}$ 内生成非荧光的加合物 $\mathbf{2 8 b}$, 荧光未发生变化, $688 \mathrm{~nm}$ 处的吸收峰降低, 产物 $\mathbf{2 8 b}$ 在 $4 \mathrm{~min}$ 内进一步与 Cys 发生置换反应生成 28a. 因此, Cys 可以置换 $\mathbf{2 8 b}$ 的 GSH 单元, 表明在一定浓度范围内 GSH 的存在不会影响探针对 Cys 的选择性.

\section{3 高半胱氨酸特异性荧光探针}

Hcy 在细胞代谢和保持生理平衡方面起着重要的作 用, 由于 Cys 和 Hcy 在分子结构上仅有一个亚甲基单元 的差异, 故一般情况下 Cys 会对 Hcy 的检测产生干扰, 因此, 开发出能对 Hcy 特异性识别的苂光探针并不容 易 ${ }^{[83]}$.

胡志强等 ${ }^{[84]}$ 基于香豆素苂光团合成了具有苂光和 比色的双通道探针 29(图 14). 由于极性溶剂会促进扭曲 的分子内电荷转移(TICT) 作用, 因此, 探针在水溶液中 几乎无荧光. 在 $\mathrm{PBS}$ 与 $\mathrm{DMSO}$ 的混合溶液 $(V / V=1 / 1, \mathrm{pH}$ $=7.4,25^{\circ} \mathrm{C}$ )中探针的吸收峰为 $513 \mathrm{~nm}$, 加入的 Hcy 能 在 $3 \mathrm{~min}$ 内与探针发生迈克尔加成反应，中断探针的共 轭结构生成 2-a, 使 $448 \mathrm{~nm}$ 处出现新的吸收峰, $513 \mathrm{~nm}$ 处的吸收峰几乎消失，同时，使苂光发射强度在 $625 \mathrm{~nm}$ 处逐渐减小, 而在 $486 \mathrm{~nm}$ 处苂光增强 8.4 倍, 检测限为 $3.5 \mu \mathrm{mol} \cdot \mathrm{L}^{-1}$. 在此过程中可以用肉眼清楚地看到溶剂 体系从紫色到黄色的颜色变化, 同时通过 486 和 
<smiles>C=CC(=O)Oc1ccc2c(c1)Oc1cc(N3CCN(C(=O)c4cc5ccc(N(CC)CC)cc5oc4=O)CC3)ccc1C21OC(=O)c2ccccc21</smiles><smiles>CCCc1c(N(CC)CC)ccc2cc(C(=O)N3CCN(c4ccc5c(-c6cccc(C(=O)O)c6)c6ccc(=O)cc-6oc5c4)CC3)c(=O)oc12</smiles>

图 11 苂光共振能量转移检测 $\mathrm{Cys}$ 的探针 $\mathbf{2 6}$

Figure 11 Probe $\mathbf{2 6}$ based on FRET for Cys detection<smiles></smiles>

27

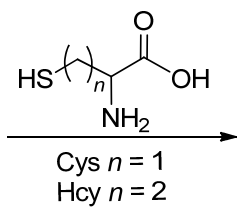

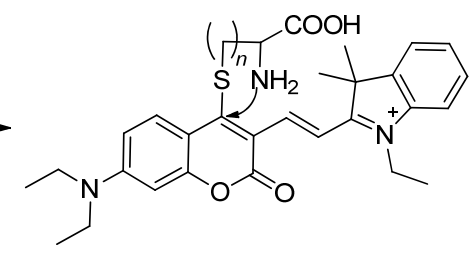

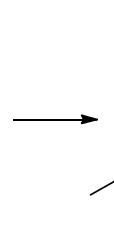<smiles>CCN(CC)c1ccc2c(NC(C)C(=O)O)c(C(Cc3c(C)c4ccccc4n3CC)C(C)(C)C)c(=O)oc2c1</smiles>

图 12 芳香取代重排检测 Cys 的探针 $\mathbf{2 7}$

Figure 12 Probe $\mathbf{2 7}$ based on aromatic substitution-rearrangement reaction for Cys detection<smiles>CN=C1C=CC2=C(Sc3ccc(OC)cc3)c3ccc(N(C)C)cc3[Si](C)(C)C2=C1</smiles>

28

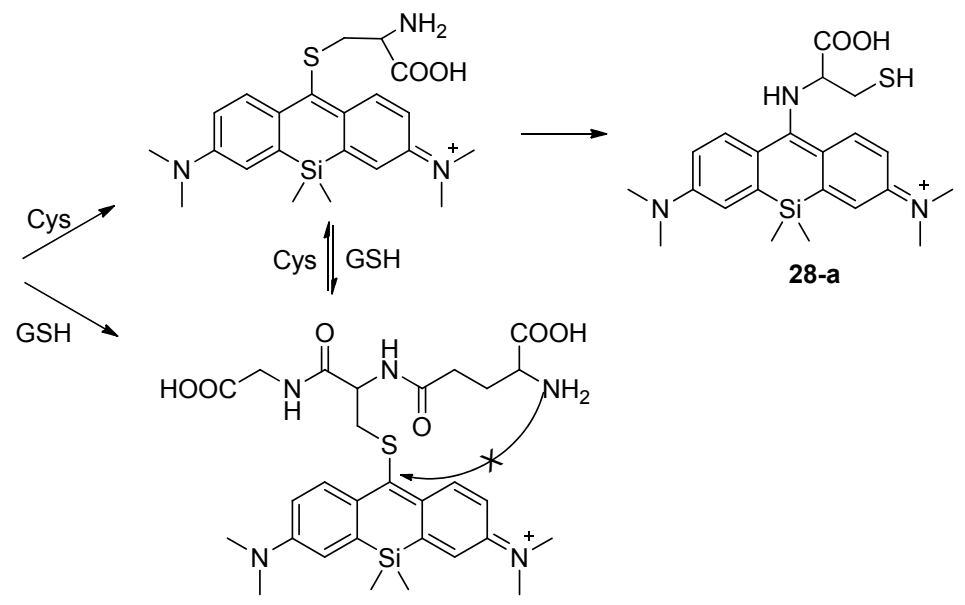

$28 b$

图 13 芳香取代重排检测 Cys 的探针 28

Figure 13 Probe $\mathbf{2 8}$ based on aromatic substitution-rearrangement reaction for Cys detection

$625 \mathrm{~nm}$ 处苂光强度的比值变化 $\left(F_{486} / F_{625}\right)$ 确定 Hcy 的最 适宜检测浓度. 其中, Cys 和 GSH 的加入只能引起微小 的吸光度和荧光强度的变化, 而其它氨基酸的加入不会 产生任何影响.

彭羽等 ${ }^{[85}$ 基于联菜骨架合成了探针 30(图 14). 在乙 醇和 HEPES $(V / V=98 / 2, \mathrm{pH}=7.0)$ 混合溶液中, Hcy 能与
探针的醛基发生环化加成反应得到产物 $\mathbf{3 0 a}$, 在 $380 \mathrm{~nm}$ 处的苂光增强 10 倍, 苂光量子产率从 $1.6 \%$ 增加至 $4.8 \%$, 对 Hcy 的检测限为 $54 \mu \mathrm{mol} \cdot \mathrm{L}^{-1}$.

解正峰等 ${ }^{[86]}$ 通过 Suzuki 偶联反应合成了具有 $\mathrm{D}-\pi-\mathrm{A}$ 结构的开启型荧光探针 31(图 14). 由于具有 ICT 和聚集诱导发射增强(AIEE)效应，探针在纯 DMF 中苂 
光很弱, 随着体系中含水比例的增加, 荧光逐步增强, 当含水量达到 $70 \%$ 时, 苂光强度值达到最大, 但当含水 量从 70\%增加到 99\%时, 聚集体的形态会发生变化, 导 致荧光自吸收现象加重, 使荧光强度又逐渐降低. 向探 针所在 DMF 与 $\mathrm{H}_{2} \mathrm{O}$ 混合溶液 $(V / V=9: 1)$ 中加入 Cys 后, 与加入 Hcy 时得到的苂光强度相比较, $I_{\mathrm{Hcy}} / I_{\mathrm{Cys}}$ 的比 值接近 19 倍, 是由于探针的酫基与 Hcy 反应生成的六 元环比与 Cys 反应得到的五元环稳定, 导致探针与 Hcy 的反应比 Cys 更快. 而 GSH 及其它氨基酸的加入则对探 针的苂光几乎没有影响. 因此, 可通过荧光强度的变化 将 Hcy 与 Cys/GSH 明显区分开来. 探针对于 Hcy 的识 别机理, 首先是 Hcy 的氨基与探针的醛基发生反应形成 亚胺中间体, 随后颈基进攻亚胺发生加成反应形成六元 环得到化合物 31a, 阻断了 ICT 效应从而使荧光增强, 发射波长为 $428 \mathrm{~nm}$, 检测限为 $0.2198 \mathrm{nmol} \cdot \mathrm{L}^{-1}$. 该探针 成功应用于活 HeLa 细胞中 Hcy 苂光成像, 为生物医学 人员探索 Hcy 在生物系统中的功能提供有效的工具.

\section{4 谷胱甘肽特异性荧光探针}

在常见的生物硫醇中, GSH 是细胞内最丰富的非蛋 白质生物硫醇, 在维持细胞内氧化还原平衡状态中起重 要作用. 由于 GSH 分子结构比其它生物硫醇大, 导致其 颈基与探针发生反应时位阻效应更明显, 且 GSH 结构 中氨基和放基距离较远, 很难像 Cys 和 Hcy一样进行氨 基和颈基的分子内取代反应 ${ }^{[87]}$. 因此, 开发出能够选择 性检测 GSH 的荧光探针具有很大的挑战.

\subsection{1 迈克尔加成反应}

通过对迈克尔受体的仔细篮选, 基于迈克尔加成反 应机理的苂光探针有望实现对 GSH 的可逆性检测. 陈 建伟等 ${ }^{[8]}$ 报道了苂光探针 32 (图 15). 在探针结构中, 氧 基可以活化相邻的双键, 增加探针的反应活性; 给体部 分引入四元杂环取代的氨基, 提高了量子产率和光稳定 性; 分子结构中的两个羧酸基团可以确保水溶性, 减少 探针与细胞内疏水性结构的结合. 探针 32 与 GSH 发生 可逆的迈克尔加成反应生成化合物 32a, 其对 GSH 的解 离常数 $\left(K_{\mathrm{d}}\right)$ 为 $3.7 \mathrm{mmol} \cdot \mathrm{L}^{-1}$, 表明该探针能够用于活细 胞内 GSH 的定量检测. 探针 32 及苂光团 32a 分别在 405 和 $488 \mathrm{~nm}$ 的激发下得到的最大发射波长分别为 487 和 $562 \mathrm{~nm}$, 通过苂光强度的比值变化 $\left(I_{405} / I_{488}\right)$ 可定量检测 GSH 的含量. 尽管 Cys/Hcy 和 GSH 对该探针具有相似 的化学反应活性, 但由于在生理条件下细胞内 Cys 水平 通常低于 $100 \mu \mathrm{mol} \cdot \mathrm{L}^{-1}$, Hcy 的含量更低, 因此 Cys/Hcy 对探针检测 GSH 的信号干扰很小. 探针可用于动态监 测细胞内因氧化还原干扰而造成的 GSH 含量变化. 同 年, Yoon 等 ${ }^{[29]}$ 合成了类似的比率型苂光探针 33 (图 16). 探针与 GSH 反应后, 苂光发射波长从 $560 \mathrm{~nm}$ 蓝移至
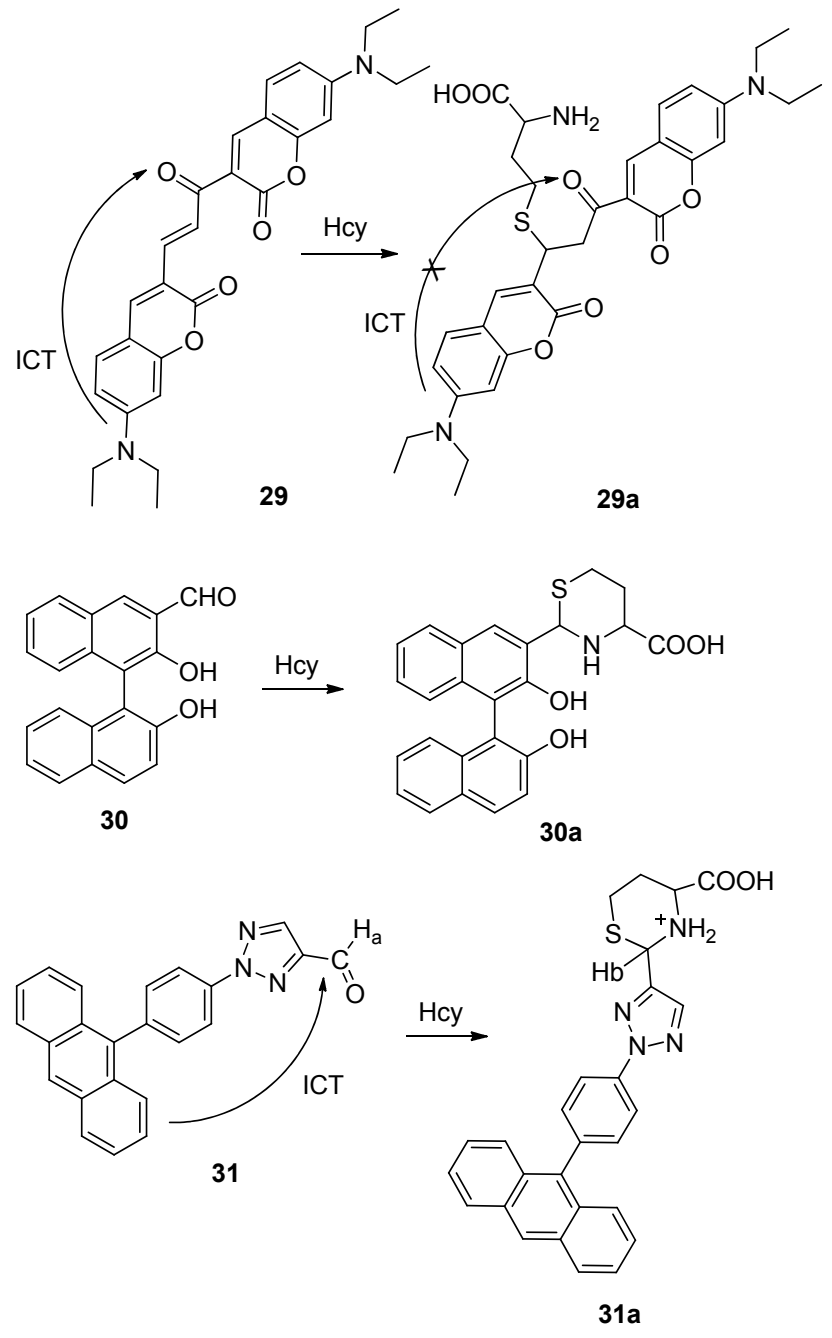

图 14 特异性检测 Hcy 的探针 29 31

Figure 14 Probes 29 31 for specific detection of Hcy

$488 \mathrm{~nm}$, 在 $450 \mathrm{~nm}$ 激发下通过苂光的比值 $\left(I_{488} / I_{560}\right)$ 可以 实现对 GSH 的定量检测. 2019 年, 郭炜 等 ${ }^{[89]}$ 利用 Rhodol 苂光团和具有 D- $\pi-A$ 结构的半花菁染料合成了 比率型荧光探针 34(图 15). 向探针溶液中加入的 GSH 能与分子中的桥连双键发生迈克尔加成反应，从而阻断 半花菁的 $\pi$ 共轭, 使 $688 \mathrm{~nm}$ 处的荧光强度逐渐减小, 在 $560 \mathrm{~nm}$ 处出现新的发射峰并逐渐增强, 以荧光强度的 比值变化 $\left(I_{560} / I_{688}\right)$ 实现对 GSH 的定量检测, 检测限为 $9.43 \mu \mathrm{mol} \cdot \mathrm{L}^{-1}$. 其中, Cys 和 Hcy 的添加仅在 $560 \mathrm{~nm}$ 处 引起较小的苂光增强, 不能对 GSH 的检测造成干扰.

\subsection{2 芳香亲核取代反应}

由于探针能与不同的生物硫醇之间发生亲核芳香 取代重排反应生成对应的硫醚，陈海燕等 ${ }^{[90]}$ 利用 2-(4羟基苯乙烯基)-4H-1-苯并吡喃-4-酮和花菁染料为荧光 团合成了可见光和 NIR 双通道荧光探针 35(图 16), 用于 选择性追踪线粒体 GSH. 探针原本在可见光区域发射 出较弱荧光, 识别 GSH 后, 颈基进攻探针的醚键, 发生 


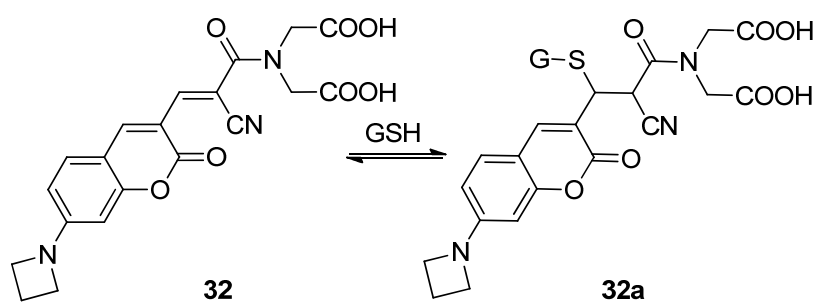

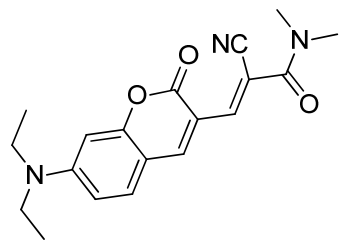

33<smiles></smiles>

34

图 15 基于 Michael 加成反应的 GSH 探针 32 34

Figure 15 GSH Probes 32 34 based on Michael addition

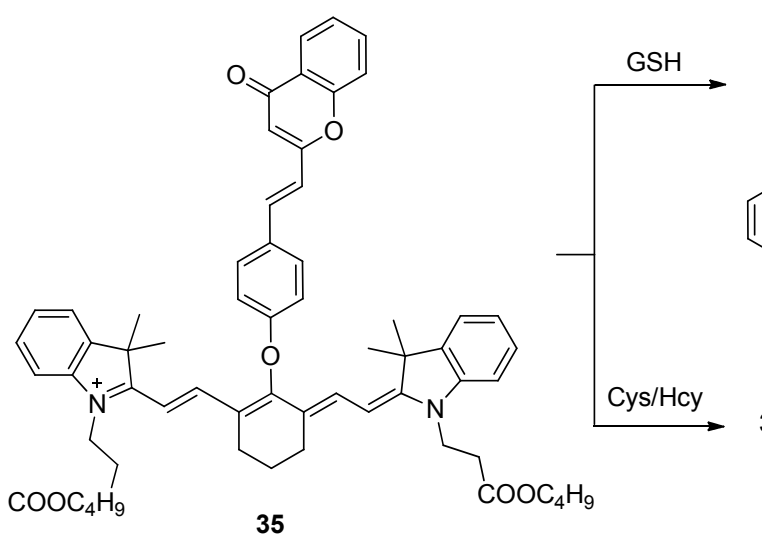

35<smiles>O=c1cc(/C=C/c2ccc(O)cc2)oc2ccccc12</smiles>

$35 a$

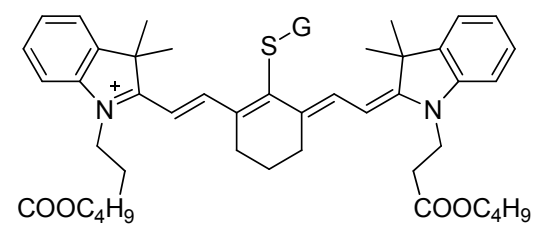

$35 b$

$35 a+$

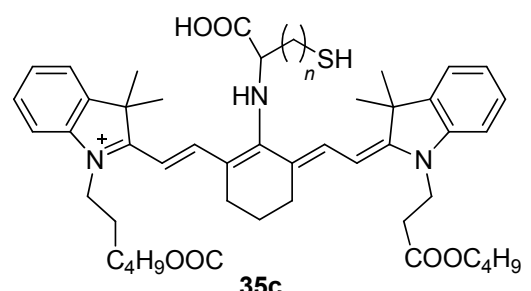

图 16 基于芳香亲核取代反应的 GSH 探针 $\mathbf{3 5}$

Figure 16 GSH probe 35 based on nucleophilic aromatic substitution

亲核芳香取代反应并释放出两个荧光团 $35 \mathrm{a}$ 和 $\mathbf{3 5} \mathbf{b}$, 发 射波长分别为 520 和 $810 \mathrm{~nm}$, 荧光强度显著增强, 检测 限分别为 24 和 $32 \mathrm{nmol} \cdot \mathrm{L}^{-1}$. Cys 和 Hcy 的氨基可以通 过 5 或 6 元环过渡态与颈基进一步交换, 形成具有不同 苂光性质的氨基取代衍生物 $\mathbf{3 5 c}$, 从而将 GSH 与 Cys/Hcy 区分开来. 同时, 探针 $\mathbf{3 5}$ 能在小鼠模型中区分 肿瘤和炎症部位, 已成功应用于 NIR 成像检测实体肿 瘤, 在临床诊断上具有广阔的应用前景.

2018 年, Yoon 等 ${ }^{[91]}$ 合成了以菜二甲酰亚胺衍生物 $(\mathrm{SNp})$ 和花菁衍生物 (CyP) 为荧光团, 以磺酰胺为识别基 团的双通道开启型苂光探针 36(图 17). 由于哌嗪环中的 叔胺氮能引起分子内单电子转移效应, 从而导致探针在 可见光区域中表现出微弱苂光, GSH 与之发生亲核取代 反应, 生成 NIR 发射产物 36a 和可见光发射产物 36b. 此过程中以 $700 \mathrm{~nm}$ 激发时, 在 $795 \mathrm{~nm}$ 处发射强苂光, 检测限为 $17.1 \mu \mathrm{mol} \cdot \mathrm{L}^{-1}$; 当以 $390 \mathrm{~nm}$ 激发时, 在 495 $\mathrm{nm}$ 处发射强荧光, 检测限为 $15.3 \mu \mathrm{mol} \cdot \mathrm{L}^{-1}$, 绿色和 NIR 荧光同时开启. 向探针溶液中加入 GSH 后, 颈基进攻磺 酰胺基团, 实验表明探针与 GSH 的反应产率比其他氨 基酸要高得多, 且探针对 GSH 在可见光和近红外通道 均具有很高的选择性. 2019 年, Yoon 等 ${ }^{[92]}$ 将吗啉引入分
子结构中, 以阳离子花菁靶向线粒体, 以含吗啉的芸酰 亚胺靶向溶酶体, 合成了具有双靶向性的 NIR 苂光探针 37(图 18). 添加的 GSH 优先攻击探针的花菁染料部分, 此过程虽然仅涉及到一个反应位点，但引发了两种不同 的亲核芳香取代反应，生成了不同的荧光发射产物 37a 和 37b (后者的生成量比前者要多), 在 $785 \mathrm{~nm}$ 处出现新 的吸收峰, 伴随溶液颜色从浅黄色变化为绿色, 并且以 $710 \mathrm{~nm}$ 为激发波长时, 在 $812 \mathrm{~nm}$ 处荧光显著增强, 检 测限为 $11 \mathrm{nmol} \cdot \mathrm{L}^{-1}$. 这将有助于开发构建单位点诱导 多反应、多发射的苂光探针.

覃文武等 ${ }^{[93]}$ 设计并合成了一种用于检测 GSH 的两 级比率型苂光探针 38(图 18). 向探针溶液中加入 GSH 后, 有两种情况: 一种是加入适量 GSH, BODIPY 上单 个氯原子被颈基取代, 生成 $\mathbf{3 8 a}$ 并伴随荧光由绿色转变 为黄色, 发射波长从 $530 \mathrm{~nm}$ 红移至 $562 \mathrm{~nm}$, 检测限为 $13.6 \mu \mathrm{mol} \cdot \mathrm{L}^{-1}$; 另一种是加入过量的 $\mathrm{GSH}$, 两个氯原子 均被颈基取代, 生成 $\mathbf{3 8 b}$ 并伴随荧光由黄色转变为红色, 发射波长从 $562 \mathrm{~nm}$ 红移至为 $597 \mathrm{~nm}$, 荧光强度比值 $\left(I_{597} / I_{562}\right)$ 超过 4.6 , 检测限为 $7.9 \mathrm{nmol} \cdot \mathrm{L}^{-1}$, 从而实现 GSH 的两步比率荧光检测, 而其他分析物在相同条件 下得到的荧光强度比值为 0.1 , 当探针溶液中添加 10 倍 


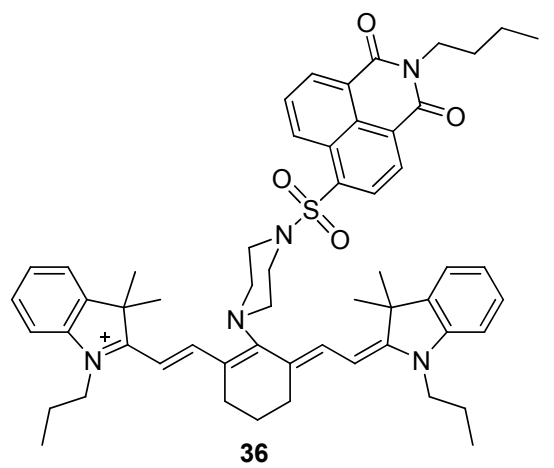<smiles>CCCN1/C(=C/C=C2\CC[B]C(/C=C/C3=[N+](CCC)c4ccccc4C3(C)C)=C2Sc2ccc3c4c(cccc24)C(=O)N(CCN2CCOC2)C3=O)C(C)(C)c2ccccc21</smiles><smiles>CCCN1/C(=C/C=C2\CCCC(/C=C/C3=[N+](CCC)c4ccccc4C3(C)C)=C2N2CCNCC2)C(C)(C)c2ccccc21</smiles>

$36 a$

$36 b$<smiles>O=C1c2cccc3c(SO)ccc(c23)C(=O)N1CCN1CCOC1</smiles>

$37 b$

图 17 基于芳香亲核取代反应的 GSH 探针 $\mathbf{3 6}$ 和 37

Figure 17 GSH Probes $\mathbf{3 6}$ and $\mathbf{3 7}$ based on nucleophilic aromatic substitution<smiles></smiles>

38<smiles></smiles>

38a<smiles>CC(C)(C)[SbH2]</smiles><smiles>F[B-]1(F)n2c(S)ccc2C(c2ccccc2)c2ccc([AsH3])n21</smiles>

38b

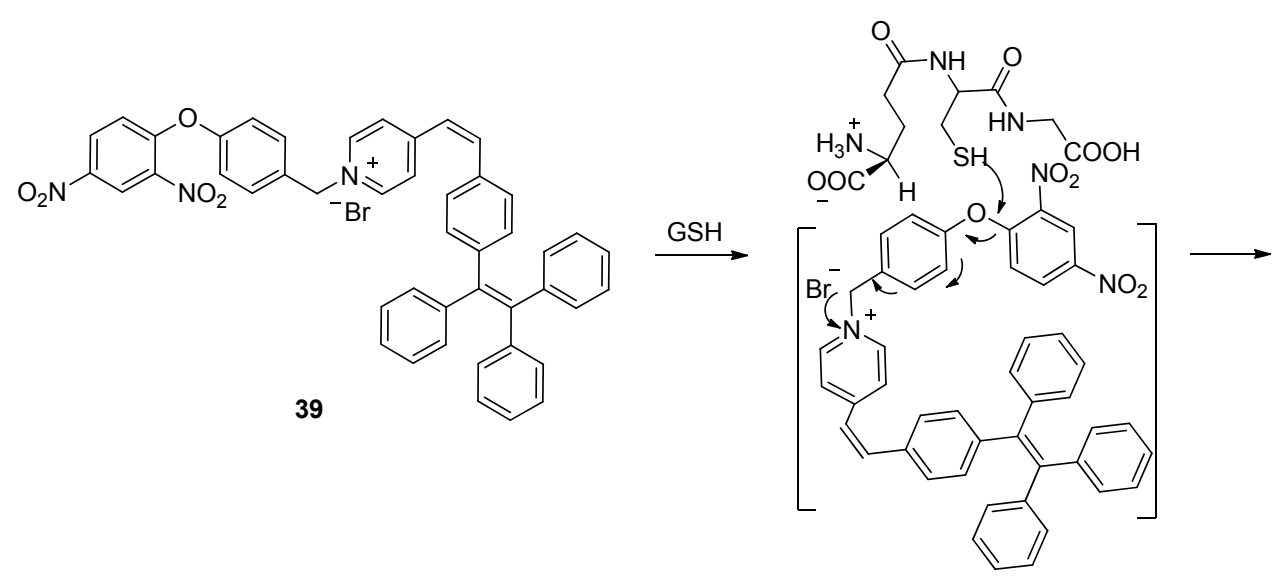<smiles>O=C1C=CC(=O)C=C1</smiles>

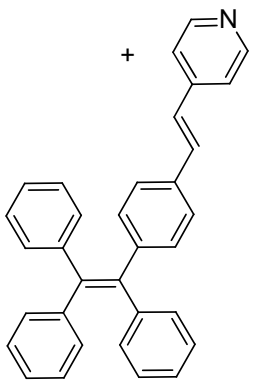

$39 a$

图 $18 \mathrm{GSH}$ 探针 $\mathbf{3 8}$ 和 39

Figure 18 GSH Probes 38 and 39

量的 Cys 和 Hcy 反应 $5 \mathrm{~min}$, 会使探针在 $530 \mathrm{~nm}$ 处的荧 光淬灭, $24 \mathrm{~h}$ 后这些荧光变化趋于稳定.

在此基础之下，唐本忠等 ${ }^{[94]}$ 报道了一种基于四苯
乙烯的双光子 AIE 比率型探针 39(图 18), 用于线粒体内 $\mathrm{GSH}$ 的检测. 在 DMSO 与 $\mathrm{H}_{2} \mathrm{O}$ 的混合体系中, 当含水 量从 0 增加到 $50 \%$ 时, 由于 TICT 效应, 探针的苂光强度 
随溶剂极性的增加而降低；当含水量从 50\%增加到 $80 \%$ 时, 由于 AIE 效应，苂光强度增加约 11 倍，发射波长从 $638 \mathrm{~nm}$ 蓝移到 $586 \mathrm{~nm}$; 而当含水量从 $80 \%$ 增加 $99 \%$ 时, 由于聚集体尺寸的变化, 苂光强度会随着波长的红移而 略微降低. 探针在 PBS 与 DMSO 的混合溶液中 $(V / V=$ $1: 1,120 \mathrm{~min}$ )发红色荧光, 加入硫醇后, 由于 $\mathrm{GSH}$ 的 分子结构能使其羧基与探针的吡啶之间相互作用, 且䘪 基能与间硝基苯酚发生亲核取代反应，再通过分子内消 除反应使羟基茮基部分脱离，从而生成了苂光团 39a, 苂光发射强度在 $500 \mathrm{~nm}$ 处增加, 在 $631 \mathrm{~nm}$ 处减少, 形 成比率型苂光响应, 检测限为 $0.61 \mu \mathrm{mol} \cdot \mathrm{L}^{-1}$. 该探针不 仅可以选择性检测线粒体内硫醇的含量水平, 还能用于 血液中硫醇的检测, 可作为早期诊断转移癌的有用工 具.

\section{2 结语与展望}

介绍了近年来生物硫醇荧光探针领域的研究进展, 以识别对象分类, 分别综述了对 Cys、Hcy 和 GSH 具有 选择性的荧光探针, 且每一类都重点介绍了不同的识别 机理, 同时对探针的分子设计、荧光变化和成像应用也 加以讨论. 可以看出, 在对硫醇的检测过程中, 已经实 现了高选择性、高灵敏度的快速检测, 并且近红外苂光 团的设计和双光子技术的引入, 使得激发波长和发射波 长大大红移，降低了生物背景干扰、减少了光致毒性、 提高了组织穿透深度, 使得成像质量显著改善. 具有聚 集诱导发光特性荧光探针的出现, 克服了传统小分子在 水溶液中容易聚集导致荧光淬灭的缺点, 为硫醇苂光探 针的开发提供了新的思路. 在众多科学家的努力下, 在 本领域已经取得进步，但是也存在一些问题：(1)硫醇探 针大多是定性或半定量检测; (2)探针在细胞内的滞留能 力和光稳定性不足, 分布不均匀; (3)细胞内三种硫醇实 际浓度差异较大导致的探针选择性问题. 为了继续解决 这些难题, 需要科研工作者的不解探索, 在此希望对已 有工作的总结, 能给未来的工作提供新的思路.

\section{References}

[1] Zhang, W.; Liu, J.; Yu, Y.; Han, Q.; Cheng, T.; Shen, J.; Wang, B.; Jiang, Y. Talanta 2018, 185, 477.

[2] Abbasi-Moayed, S.; Golmohammadi, H.; Bigdeli, A.; HormoziNezhad, M. R. Analyst 2018, 143, 3415.

[3] Chen, D.; Yang, J.; Dai, J.; Lou, X.; Zhong, C.; Yu, X.; Xia, F. J. Mater. Chem. B 2018, 6, 5248.

[4] Alberti, G.; Nurchi, V. M.; Magnaghi, L. R.; Biesuz, R. Anal. Methods 2019, 11, 4464.

[5] Yang, Y.; Wang, Y.; Feng, Y.; Cao, C.; Song, X.; Zhang, G.; Liu, W. J. Mater. Chem. B 2019, 7, 7723.

[6] Zhan, C.; Zhang, G.; Zhang, D. ACS Appl. Mater. Interfaces 2017, $10,12141$.

[7] Jiao, S.; He, X.; Xu, L.; Ma, P.; Liu, C.; Huang, Y.; Sun, Y.; Wang,
X.; Song, D. Sens. Actuators, B 2019, 290, 47.

[8] Li, M.; Kang, N.; Zhang, C.; Liang, W.; Zhang, G.; Jia, J.; Yao, Q.; Shuang, S.; Dong, C. Spectrochim. Acta, Part A 2019, 222, 117262.

[9] Wu, Q.; Mao, M.; Liang, W.; Stadler, F. J. Talanta 2018, 186, 110.

[10] Yin, C. X.; Xiong, K. M.; Huo, F. J.; Salamanca, J. C.; Strongin, R. M. Angew. Chem. Int. Ed. 2017, 56, 13188.

[11] Niu, H.; Ni, B.; Chen, K.; Yang, X.; Cao, W.; Ye, Y.; Zhao, Y. Talanta 2019, 196, 145.

[12] Kim, C. Y.; Kang, H. J.; Chung, S. J.; Kim, H. K.; Na, S. Y.; Kim, H. J. Anal. Chem 2016, 88, 7178.

[13] Wang, H.; Zhang, Y.; Yang, Y.; He, Z.; Wu, C.; Zhang, W.; Zhang, W.; Liu, J.; Li, P.; Tang, B. Chem. Commun. 2019, 55, 9685.

[14] Bai, Z.; Yan, F.; Xu, J.; Zhang, J.; Wei, J.; Luo, Y.; Chen, L. Spectrochim. Acta, Part A 2018, 205, 29.

[15] Yue, Y.; Huo, F.; Ning, P.; Zhang, Y.; Chao, J.; Meng, X.; Yin, C. J. Am. Chem. Soc. 2017, 139, 3181.

[16] Yang, M. W.; Fan, J. L.; Sun, W.; Du, J. J.; Peng, X. J. Anal. Chem. 2019, 19, 12531

[17] Li, M.; Zheng, K.; Chen, H.; Liu, X.; Xiao, S.; Yan, J.; Tan, X.; Zhang, N. Spectrochim. Acta, Part A 2019, 217, 1.

[18] Meng, X.; Ye, W.; Wang, S.; Feng, Y.; Chen, M.; Zhu, M.; Guo, Q. Sens. Actuators, $B$ 2014, 201, 520.

[19] Zhang, Q.; Cui, Z.; Wang, Q.; Zheng, G. Sens. Actuators, B 2019, 295,79

[20] Guo, Z. B.; Zheng, X. Y.; Li, X. Y.; Jia, Q. F.; Zhang, P. Z.; Wei, C.; Li, X. L. Chin. J. Org. Chem. 2020, 40, 1239 (in Chinese). (郭振波, 郑雪阳, 李雪艳, 贾清菲, 张平竹, 魏超, 李小六, 有 机化学, 2020, 40, 1239.)

[21] Chen, D.; Zhong, C.; Zhao, Y.; Nan, L.; Liu, Y.; Qin, J. J. Mater. Chem. C 2017, 5, 5199.

[22] Li, D.-P.; Zhang, J.-F.; Cui, J.; Ma, X.-F.; Liu, J.-T.; Miao, J.-Y.; Zhao, B.-X. Sens. Actuators, B 2016, 234, 231.

[23] Zhang, Y.; Wang, X.; Bai, X.; Li, P.; Su, D.; Zhang, W.; Zhang, W.; Tang, B. Anal. Chem. 2019, 91, 8591.

[24] Dang, Y.; Chen, L.; Yuan, L.; Li, J.; Chen, D. ChemistrySelect 2020, 5,584

[25] Yan, P. P.; Wang, T.; Zhang, D.; Ma, X. X. Chin. J. Org. Chem. 2019, 39, 916 (in Chinese) (闵沛沛，王婷，张丹，马晓雪，有机化学，2019, 39, 916.)

[26] Chen, H.; Tang, Y.; Lin, W. Trends Anal. Chem. 2016, 76, 166.

[27] Chao, J.; Li, M.; Liu, Y.; Zhang, Y.; Huo, F.; Yin, C. Sens. Actuators, B 2019, 298, 126844.

[28] Huang, Y.; Mei, J.; Ma, X. Dyes Pigm. 2019, 165, 499.

[29] Liu, Z.; Zhou, X.; Miao, Y.; Hu, Y.; Kwon, N.; Wu, X.; Yoon, J. Angew. Chem. Int. Ed. 2017, 56, 5812.

[30] Yang, T.; Niu, D.; Chen, J.; He, J.; Yang, S.; Jia, X.; Hao, J.; Zhao, W.; Li, Y. Biomater. Sci. 2019, 7, 2951.

[31] Wang, L.; Wu, S.; Tang, H.; Meier, H.; Cao, D. Sens. Actuators, B 2018, 273, 1085 .

[32] Long, Z.; Chen, L.; Dang, Y.; Chen, D.; Lou, X.; Xia, F. Talanta 2019, 204, 762.

[33] Zhou, T. T.; Yang, Y. T.; Zhou, K. Y.; Xu, W. Z.; Li, W. Chin. J. Org. Chem. 2019, 39, 3498 (in Chinese). (周婷婷, 杨瑜涛, 周柯岩, 胥稳智, 李玮, 有机化学, 2019, 39, 3498.)

[34] Nia, N. N.; Hadjmohammadi, M. R. Anal. Methods 2019, 11, 5134.

[35] Cao, L.; Liang, Q.; Wei, T.; Shi, Y.; Deng, T.; Meng, J. J. Chromatogr. A 2018, 1577, 47.

[36] Zhang, Y.; Zhang, Y.; Zhu, L.; He, P.; Wang, Q. Anal. Methods 2019, 11, 1558 .

[37] Otto, P.; Bergmann, S.; Sandmeyer, A.; Dirksen, M.; Wrede, O.; Hellweg, T.; Huser, T. Nanoscale Adv. 2020.

[38] Zhang, Y. N.; Niu, Q.; Gu, X.; Yang, N.; Zhao, G. Nanoscale 2019, $11,11992$.

[39] Hagemann, L. T.; McCartney, M. M.; Fung, A. G.; Peirano, D. J.; Davis, C. E.; Mizaikoff, B. Analyst 2018, 143, 5683.

[40] Jiang, T.; Wang, X.; Wang, G.; Wang, Y. F.; Wang, K.; Xuan, X. P.; Chen, C. P.; Jiang, K.; Zhang, H. Chem. Commun. 2019, 55, 5279. 
[41] Li, R.; Huang, X.; Lu, G.; Feng, C. RSC Adv. 2018, 8, 24346.

[42] Dai, X.; Zhang, T.; Miao, J.-Y.; Zhao, B.-X. Sens. Actuators, B 2016, 223, 274.

[43] Ramachandran, R.; Chen, T.-W.; Chen, S.-M.; Baskar, T.; Kannan, R.; Elumalai, P.; Raja, P.; Jeyapragasam, T.; Dinakaran, K.; Gnana kumar, G. p. Inorg. Chem. Front. 2019, 6, 3418.

[44] Chen, K.; Han, B. C.; Ji, S. X.; Sun, J.; Gao, Z. Z.; Hou, X. F. Acta Chim. Sinica 2019, 77, 365 (in Chinese). (陈凯, 韩百川, 森思金金, 孙瑾, 高振忠, 侯贤锋, 化学学报, 2019, 77, 365.)

[45] Xie, C.; Ma, C.; Jia, X.; Zhang, X. Q.; Wei, C.; Zhang, P. Z.; Li, X. L. Chin. J. Org. Chem. 2019, 39, 3277 (in Chinese). (解畅, 马趁, 贾旭, 张学琪, 魏超, 张平竹, 李小六, 有机化学, 2019, 39, 3277.)

[46] Liu, H.; Zhu, L.; Lou, X.; Yuan, L.; Zhang, X. Acta Chim. Sinica 2020, 78, 1240 (in Chinese).

(刘红文, 朱隆民, 娄霄峰, 袁林, 张晓兵, 化学学报, 2020, 78, 1240.)

[47] Zhang, J. D.; Liu, A. C.; Chen, J.; Yuan, G. H.; Jin, H. F. Prog. Chem. 2020, 32, 594 (in Chinese).

(张继东, 刘阿晨, 陈娇, 袁光辉, 金华峰, 化学进展, 2020, 32, 594.)

[48] Liu, Y.; Niu, L.-Y.; Chen, Y.-Z.; Yang, Q.-Z. J. Photochem. Photobiol. A, Chem. 2018, 355, 311.

[49] Wang, Y.; Huang, C. S.; Jia, N. Q. Prog. Chem. 2019, 32, 204 (in Chinese). (王阳, 黄楚森, 贾能勤, 化学进展, 2019, 32, 204.)

[50] Zhu, D.; Yan, X.; Ren, A.; Xie, W.; Duan, Z. Anal. Chim. Acta 2019, 1058, 136.

[51] Chen, D.; Long, Z.; Sun, Y.; Luo, Z.; Lou, X. J. Photochem. Photobiol. A, Chem. 2019, 368, 90.

[52] Yang, L.; Xiong, H.; Su, Y.; Tian, H.; Liu, X.; Song, X. Chin. Chem. Lett. 2019, 30, 563.

[53] Du, W.; Liu, R.; Fang, J.; Gao, H.; Wang, Y.; Peng, Y. Tetrahedron 2019, 75, 130477.

[54] Huang, Y.; Zhang, Y.; Huo, F.; Liu, Y.; Yin, C. Sens. Actuators, B 2019, 301, 127123.

[55] Luo, J.; Xie, Z.; Lam, J. W. Y.; Cheng, L.; Chen, H.; Qiu, C.; Kwok, H. S.; Zhan, X.; Liu, Y.; Zhu, D.; Tang, B. Z. Chem. Commun. 2001, 18, 1740 .

[56] Mei, J.; Leung, N. L. C.; Kwok, R. T. K.; Lam, J. W. Y.; Tang, B. Z. Chem. Rev. 2015, 115, 11718.

[57] Dong, F.; Lai, H.; Liu, Y.; Li, Q.; Chen, H.; Ji, S.; Zhang, J.; Huo, Y. Talanta 2020, 206, 120177.

[58] Das, S.; Ghosh, A.; Kundu, S.; Saha, S.; Sarkar, H. S.; Sahoo, P. Anal. Bioanal. Chem. 2019, 411, 6203.

[59] Huang, Z.; Wu, C.; Li, Y.; Zhou, Z.; Xie, R.; Pang, X.; Xu, H.; Li, H.; Zhang, Y. Anal. Methods 2019, 11, 3280.

[60] Wang, B. J.; Liu, R. J.; Fang, J. G.; Wang, Y. W.; Peng, Y. Chem. Commun. 2019, 55, 11762.

[61] Ning, Z. W.; Wu, S. Z.; Liu, G. J.; Ji, Y. M.; Jia, L. Y.; Niu, X. X.; Ma, R. F.; Zhang, Y.; Xing, G. W. Chem. Asian J. 2019, 14, 2220.

[62] Khatik, G. L.; Kumar, R.; Chakraborti, A. K. Org. Lett. 2006, 8, 2433.

[63] Lin, W.; Yuan, L.; Cao, Z.; Feng, Y.; Long, L. Chem.-Eur. J. 2009, $15,5096$.

[64] Zhou, X.; Jin, X.; Sun, G.; Li, D.; Wu, X. Chem. Commun. 2012, $48,8793$.

[65] Zhao, N.; Wu, Y. H.; Shi, L. X.; Lin, Q. P.; Chen, Z. N. Dalton
Trans. 2010, 39, 8288

[66] Li, Y.; Shi, N.; Li, M. New J. Chem. 2019, 43, 18517.

[67] Chen, X.; Xu, H.; Ma, S.; Tong, H.; Lou, K.; Wang, W. RSC Adv. 2018, 8, 13388.

[68] Zhu, M.; Wu, X.; Sang, L.; Fan, F.; Wang, L.; Wu, X.; Hua, R.; Wang, Y.; Li, Q. X. New J. Chem. 2019, 43, 13463.

[69] Zhang, P.; Xiao, Y.; Zhang, Q.; Zhang, Z.; Yu, H.; Ding, C. New J. Chem. 2019, 43, 7620 .

[70] Han, C.; Song, B.; Liang, X.; Pan, H.; Dong, W. Anal. Methods 2019, 11, 2513.

[71] Wu, Y.; Shi, A.; Zeng, H.; Li, Y.; Li, H.; Chen, X.; Wong, W.-Y.; Fan, X. Dyes Pigm. 2019, 170, 107563.

[72] Lu, Z.; Lu, Y.; Fan, C.; Sun, X.; Shao, W.; Jiang, N.; Gong, X.; Lu, Y.; Sun, G.; Jiang, X. Sens. Actuators, B 2019, 290, 581.

[73] Zhang, S.; Wu, D.; Wu, J.; Xia, Q.; Jia, X.; Song, X.; Zeng, L.; Yuan, Y. Talanta 2019, 204, 747.

[74] Yu, Y.; Xu, H.; Zhang, W.; Han, Q.; Wang, B.; Jiang, Y. J. Photochem. Photobiol. A, Chem 2017, 346, 215.

[75] Dai, Y.; Xue, T.; Zhang, X.; Misal, S.; Ji, H.; Qi, Z. Spectrochim. Acta, Part A 2019, 216, 365

[76] Liu, D.; Lv, Y.; Chen, M.; Cheng, D.; Song, Z.; Yuan, L.; Zhang, X. J. Mater. Chem. B 2019, 7, 3970 .

[77] Chen, D.; Long, Z.; Dang, Y.; Chen, L. Dyes Pigm. 2019, 166, 266.

[78] Liang, B.; Wang, B.; Ma, Q.; Xie, C.; Li, X.; Wang, S. Spectrochim. Acta, Part A 2018, 192, 67.

[79] Bai, Y.; Wu, M.-X.; Ma, Q.-J.; Wang, C.-Y.; Sun, J.-G.; Tian, M.-J.; Li, J.-S. New J. Chem. 2019, 43, 14763.

[80] Kang, Y.-F.; Niu, L.-Y.; Yang, Q.-Z. Chin. Chem. Lett. 2019, 30, 1791.

[81] Yang, Y.; Wang, H.; Wei, Y.-L.; Zhou, J.; Zhang, J.-F.; Zhou, Y. Chin. Chem. Lett. 2017, 28, 2023.

[82] Liu, J.; Liu, M.; Zhang, H.; Wei, X.; Wang, J.; Xian, M.; Guo, W. Chem. Sci. 2019, 10, 10065.

[83] Chen, X.; Wang, F.; Hyun, J. Y.; Wei, T.; Qiang, J.; Ren, X.; Shin, I.; Yoon, J. Chem. Soc. Rev. 2016, 45, 2976.

[84] Wang, K.; Xu, S.; Lei, Y.; Zheng, W.; Zhang, Q.; Chen, S.; Hu, H.; $\mathrm{Hu}, \mathrm{Z}$. Talanta 2019, 196, 243.

[85] Wang, Y. W.; Liu, S. B.; Ling, W. J.; Peng, Y. Chem. Commun. 2016, 52,827

[86] Chu, Y.; Xie, Z.; Zhuang, D.; Yue, Y.; Yue, Y.; Shi, W.; Feng, S. Chin. J. Chem. 2019, 37, 1216.

[87] Wang, C.; Xia, X.; Luo, J.; Qian, Y. Dyes Pigm. 2018, 152, 85.

[88] Jiang, X.; Chen, J.; Bajic, A.; Zhang, C.; Song, X.; Carroll, S. L.; Cai, Z. L.; Tang, M.; Xue, M.; Cheng, N.; Schaaf, C. P.; Li, F.; MacKenzie, K. R.; Ferreon, A. C. M.; Xia, F.; Wang, M. C.; Maletic-Savatic, M.; Wang, J. Nat. Commun. 2017, 8, 16087.

[89] Ren, M.; Wang, L.; Lv, X.; Sun, Y.; Chen, H.; Zhang, K.; Wu, Q.; Bai, Y.; Guo, W. Analyst 2019, 144, 7457.

[90] Xu, Y.; Li, R.; Zhou, X.; Li, W.; Ernest, U.; Wan, H.; Li, L.; Chen, H.; Yuan, Z. Talanta 2019, 205, 120125.

[91] Xu, Z.; Huang, X.; Han, X.; Wu, D.; Zhang, B.; Tan, Y.; Cao, M.; Liu, S. H.; Yin, J.; Yoon, J. Chem. 2018, 4, 1609.

[92] Xu, Z.; Zhang, M.-X.; Xu, Y.; Liu, S. H.; Zeng, L.; Chen, H.; Yin, J. Sens. Actuators, B 2019, 290, 676.

[93] Gong, D.; Ru, J.; Cao, T.; Qian, J.; Liu, W.; Iqbal, A.; Liu, W.; Qin, W.; Guo, H. Sens. Actuators, B 2018, 258, 72.

[94] Gu, Y.; Zhao, Z.; Niu, G.; Zhang, R.; Zhang, H.; Shan, G.-G.; Feng, H.-T.; Kwok, R. T. K.; Lam, J. W. Y.; Yu, X.; Tang, B. Z. ACS Appl. Bio Mater. 2019, 2, 3120 